\title{
Lamellae Stability in Confined Systems with Gravity
}

\author{
M. Bahiana, W. A. M. Morgado \\ Instituto de Física, UFRJ \\ Rio de Janeiro, RJ, Brazil
}

(April 18, 2017)

\begin{abstract}
The microphase separation of a diblock copolymer melt confined by hard walls and in the presence of a gravitational field is simulated by means of a cell dynamical system model. It is found that the presence of hard walls normal to the gravitational field are key ingredients to the formation of well ordered lamellae in BCP melts. To this effect the currents in the directions normal and parallel to the field are calculated along the interface of a lamellar domain, showing that the formation of lamellae parallel to the hard boundaries and normal to the field correspond to the stable configuration. Also, it is found thet the field increases the interface width.
\end{abstract}

64.60.Cn,61.41.+e,64.75.+g

Our main motivation for the present work has been to get insight about the mechanism responsible for the formation of well ordered layered samples of diblock copolymers, but, since the same striped pattern is observed in a variety of systems (zebra skin, fingerprints, and the visual cortex, to mention some), we expect our results to be useful for the general problem of pattern formation in finite systems. The formation of the striped pattern has been studied by several authors and some factors important to the phenomenon have already been identified: (i) hydrodynamic interactions combined with the preferential wetting of a surface [1]; (ii) annealing and quenching of the structure, which are capable of relaxing the frustrated structure locally 2;;(iii) long range interactions with an external surface [3].

We intend to study the influence of two different effects that have not been included in the references quoted above (and still need to be better understood in the general context of block copolymers): the simultaneous presence of the gravitational field and of a rigid wall limiting the actual size of the sample, as in realistic systems. For that matter we consider simulations of a block copolymer (BCP) melt based on a cell dynamical system (CDS) model [4] and the modified Cahn-Hilliard ( $\mathrm{CH}$ ) equation for driven spinodal decomposition [5]. In both cases we analyze systems with a gravitational field normal to the hard boundary with the intention of reproducing the presence of a substrate. As observed in real systems, the layers are formed parallel to the substrate, even in the absence of interaction with the wall.

Block copolymers are linear-chain molecules consisting of two each other. The subchains $a$ and $b$ are made of different monomer units, $A$ and $B$, respectively. Below some critical temperature $T_{c}$ these two blocks tend to separate, but due to the covalent bond, they can segregate at best locally to form a lamellar structure. The description of the microphase separation is similar to the spinodal decomposition of binary mixtures [1, 6], so we borrow the CDS model proposed by Kitahara et al for the spinodal decomposition with an external force field with the appropriate addition of a term that makes the large uniform domains unstable. As usual, we assign a scalar variable $\psi(n, t)$ to each lattice site corresponding to the coarse-grained order parameter in the $n$-th cell at time $t$ (time here is defined as the number of iterations). This order parameter represents the difference $\psi_{A}-\psi_{B}$, where $\psi_{A}\left(\psi_{B}\right)$ is the local number density of $A(B)$. The ingredients for the time evolution of $\psi$ are: local dynamics dictated by a function with two symmetric hyperbolic attractive fixed points, diffusive coupling with neighbors, stabilization of the homogeneous solution and conservation of $\psi$. The conservation, when an external field is present, must be imposed by considering the Kawasaki exchange dynamics explicitly. The detailed explanation of this model is found in 画 for spinodal decomposition. With this, we come to final equation for a melt of even BCP molecules :

$$
\psi(n, t+1)=(1-B) \psi(n, t)+\langle\langle C(i, j ; \operatorname{sgn}[I(n, t)-I(j, t)])(I(n, t)-I(j, t))\rangle\rangle,
$$

where

$$
I(n, t) \equiv A \tanh (\psi(n, t))-\psi(n, t)+D(\langle\langle\psi(n, t)\rangle\rangle-\psi(n, t))-h n_{z}
$$

is essentially the chemical potential. $\langle\langle\star\rangle\rangle$ is the isotropic space average of $\star, B, A$ and $D$ are positive phenomenological constants. The parameter $B$ appears in this model to stabilize the solution $\psi=0$ in the bulk, for $B=0$ we have a model for spinodal decomposition, in which the domains can grow without bound. Scaling arguments have proved that $B \sim N^{-2}$, where $N$ is the polymerization index ( [7]). $h$ is the external field, which we assume is in the $z$ direction, and $n_{z}$ is the $z$ component of $n$. The collision coefficient is given by: $C(i, j ; \alpha)=\left[\psi_{c}+\alpha \psi(j)\right]\left[\psi_{c}-\alpha \psi(i)\right] / \psi_{c}^{2}$, where $\pm \psi_{c}$ are the fixed points of $A \tanh \psi-\psi$. 
For all the simulations we used a $128 \times 256$ lattice, $A=1.2, D=0.5$ and uniformly distributed random initial conditions. The external field, when present, is parallel to the smaller dimension, which we call $z$. The direction normal to the field will be called the $x$ direction. We impose no flux boundary conditions: $I(z+1)-\left.I(z)\right|_{\text {boundaries }}=0$. Figure 1 shows the pattern obtained after 20,000, 40,000 and 60,000 iterations, for $\mathrm{B}=0.018$ and $\mathrm{h}=0.001$.

Domains initially aligned with the field present a varicose instability [8] triggered by a density increase near the hard boundaries. In this varicose mode the bulges tend to grow making the coalescence of the domains possible and we observe the formation of lamellae on the "substrate". The central region, oriented along the field, shrinks with time. It is interesting to compare these patterns to the ones obtained by Brown and Chakrabarti in [3]. These authors numerically integrate the Cahn-Hilliard like equation of motion for the microphase separation dynamics with an extra term in the free energy functional that takes into account a long-range interaction with a substrate. What they see is truly a surface effect, their patterns present two regions: one with ordered lamellae, and another one with randomly oriented lamellae. That same pattern had already been reported in [1] with a much simpler boundary condition: just keeping one line with a fixed value of $\psi$. Here we observe patterns with regions where the lamellae are oriented normally to the field on top and bottom, ordered such that the denser part is in contact with the substrate, and another one in which the lamellae prefer to be parallel to the field, as found in systems with full periodic boundary conditions [9]. For the same boundary condition and $h=0$, no layers are formed. To conclude the comparison, we have performed simulations using a CDS model with the same form of surface field as in [3] and no bulk field to be compared to simulations with the bulk field only. Adjusting the value of $h$ such that the boundaries in both cases have the same values of the field, we need a surface field one order of magnitude larger to obtain the same number of lamellae. The patterns with the surface and bulk field are shown in Figure 2

The presence of the field does not affect the linear stability about the homogeneous state $\psi=0$. We find that the solution $\psi=0$ is unstable when $B \leq(A-1)^{2} / 4 D$, independently of the field. For the values $A=1.2$ and $D=0.5$ used in the simulations here, we should expect to observe pattern formation for $B \leq 0.1$. What we actually see from the simulations is quite different, though. For example, for $B=0.018, \psi=0$ is stable for $h>0.02$. For smaller fields, there is pattern formation, but the amplitude of $\psi$ is smaller than that measured in the $h=0$ simulations. The decrease in amplitude is accompanied by an increase in the interface width, so the resulting pattern is less segregated than that without the field. As for the domain morphology the early stages of microphase separation are similar in driven and non-driven systems [9], in agreement with the linear stability analysis.

To understand the formation of lamellae on the hard boundaries we analyze the stability of one vertical lamella in the presence of a gravitational field. It is well known that a gravitational field enhances the stability of interfaces parallel to itself [5] which is not observed near the hard boundary. To understand this we assume that close to the interface, the non-local term of the $\mathrm{CH}$ free energy for block copolymers is an irrelevant constant. In this spirit, we use the modified $\mathrm{CH}$ equation proposed in 5 for the spinodal decomposition in the presence of a gravitational field in the $z$-direction:

$$
\frac{\partial \psi}{\partial t}=\nabla \cdot\left[\left(1-\frac{\psi^{2}}{\psi_{c}}\right)\left(\nabla \frac{\delta F}{\delta \psi}-h \hat{z}\right)\right]
$$

where $F[\psi]$ is the usual CH free energy, $F=\int d \mathbf{r}\left(\frac{1}{2} k(\nabla \psi)^{2}-\frac{1}{2} r_{0} \psi^{2}+\frac{1}{4} g \psi^{4}\right)$, for binary systems. The steady state solutions of (3) for a system with hard walls normal to the field satisfy

$$
\begin{aligned}
& j_{x}=-\partial_{x}\left(k \partial_{x}^{2} \psi+r_{0} \psi-g \psi^{3}\right)-k \partial_{z}^{2} \partial_{x} \psi=0 \\
& j_{z}=-\partial_{z}\left(k \partial_{z}^{2} \psi+r_{0} \psi-g \psi^{3}\right)-k \partial_{x}^{2} \partial_{z} \psi=h
\end{aligned}
$$

With this, we see that the presence of the bottom wall forbids the stable flux in the vertical direction so the system seeks the naturally stable pattern, the one parallel to the wall. From (4b) it can be shown, for the case of horizontal lamellae, that the interface becomes less sharp with increasing $h$. This effect is noticeable in Fig. 2: all the patterns where plotted with the same grey scale so it is clear that pattern (b), with gravitational field, is more diffuse. This result can be understood if we assume that $\psi(z)=\psi_{e} \tanh (z / \xi)+\epsilon \psi_{1}+O\left(h^{2}\right)$ for a particular interface located at $z=0$, where $\psi_{e}=\sqrt{r_{0} / g}, \xi=\sqrt{2 k / r_{0}}$ and $\epsilon=h / k$. We obtain $\psi_{1}=-\frac{z}{4 \xi} \psi_{e}$, hence

$$
\psi(z)=\psi_{e}\left(\tanh \frac{z}{\xi}-\epsilon \frac{z}{4 \xi}\right)
$$

and 


$$
\begin{aligned}
& \psi_{h=0}^{\prime}(0)=\frac{\psi_{e}}{\xi} \\
& \psi_{h \neq 0}^{\prime}(0)=\frac{\psi_{e}}{\xi}\left(1-\frac{\epsilon}{4}\right)<\frac{\psi_{e}}{\xi},
\end{aligned}
$$

which shows that the interface has an elongated profile in the presence of the field. In summary, we conclude that the presence of hard walls normal to the gravitational field are key ingredients to the formation of well ordered lamellae in BCP melts. Other interactions like hydrodynamics and preferential wetting of the substrate may enhance this effect, but we find that the bulk field considered here is more efficient than a surface field for ordering the lamellae, and involves a different mechanism of lamellae formation. We also find that the gravitational field increases the interface width, producing less segrgated patterns.

\section{ACKNOWLEDGMENTS}

This work was partially supported by CNPq (Brazil) and Faperj (state of Rio de Janeiro, Brazil). The simulations were done on the Cray-J90 computer of the NACAD, COPPE/UFRJ, Brazil.

[1] M. Bahiana and Y. Oono, Phys. Rev. A 41, 6763 (1990).

[2] A. Chakrabarti and J. D. Gunton, Phys. Rev. E 47, R792 (1993).

[3] G.Brown and A.Chakrabarti, J. Chem. Phys. 101, 3310 (1994).

[4] K. Kitahara, Y. Oono, and D. Jasnow, Mod. Phys. Lett. B 2, 765 (1988).

[5] C. Yeung, T. Rogers, A. Hernandez-Machado, and D. Jasnow, J. Stat. Phys 66, 1071 (1992).

[6] Y. Oono and Y. Shiwa, Mod. Phys. Lett. B 1, 49 (1987).

[7] Y. Oono and M. Bahiana, Phys. Rev. Lett. 61, 1109 (1988).

[8] A.Frischknecht, Phys. Rev. E 56, 6970 (1997).

[9] M. Bahiana, to be published in Physica A.

FIG. 1. Simulation of a driven system with no flux boundary conditions. Here, $A=1.2, B=0.018$ and $h=0.001$ after (a) 5,000 , (b) 20,000 and (c) 60,000 iterations. Lamellae oriented normal to the external field accumulate at the hard boundaries, as observed in real systems.

FIG. 2. Here we compare the long-range surface interaction of the form $h_{s} / z^{3}$ as proposed by Brown and Chakrabarti. For all three patterns $B=0.018$. In (a) is the pattern obtained with surface interaction only and $h_{s}=0.001$. (b) corresponds to the pattern with gravitational field $h=0.001$, and (c) surface field $h_{s}=0.01$. For the values chosen, (a) and (b) have the same field at $z=1$, but the pattern with gravity is more ordered. Close to the substrate patterns (b) and (c) present the same number of 1 amellae but the filed in (c) is ten times larger, showing that the bulk field is more effective in producing well ordered lamellae. 